\title{
Preexisting Right Ventricular Systolic Dysfunction May Be More Prognostic Than Left Ventricular Systolic Dysfunction for Mortality in High-Risk Patients Undergoing Non-Emergent Open Abdominal Surgery: A Retrospective Cohort Study
}

Jody Chou ( $\nabla$ cjchou@uci.edu )

University of California Irvine https://orcid.org/0000-0002-6462-458X

Michael Ma

University of California Irvine

Maryte Gylys

University of California Irvine

Nicolas Salvatierra

University of California Irvine

Robert Kim

University of California Irvine

Luohua Jiang

University of California Irvine

Ailin Barseghian

University of California Irvine

Joseph Rinehart

University of California Irvine

\section{Research Article}

Keywords: Preexisting right ventricular systolic dysfunction, open abdominal surgery, major adverse cardiac events, mortality

Posted Date: February 13th, 2019

DOI: https://doi.org/10.21203/rs.2.330/v1

License: (c) (1) This work is licensed under a Creative Commons Attribution 4.0 International License.

Read Full License 


\section{Abstract}

Background: The prognostic value of right ventricular systolic dysfunction in high-risk patients undergoing non-emergent open abdominal surgery is unknown. Here, we aim to evaluate whether presence of preexisting right ventricular systolic dysfunction in this surgical cohort is independently associated with higher incidence of postoperative major adverse cardiac events and all-cause in-hospital mortality.

Methods: This is a single-centered retrospective study. Patients identified as American Society Anesthesiology Classification III and IV who had a preoperative echocardiogram within 1 year of undergoing non-emergent open abdominal surgery between January 2010 and May 2017 were included in the study. Incidence of postoperative major cardiac adverse events and all-cause in-hospital mortality were collected. Multivariable logistic regression was performed in a step-wise manner to identify independent association between preexisting right ventricular dysfunction with outcomes of interest.

Results: Preexisting right ventricular systolic dysfunction was not associated with postoperative major adverse cardiac events $(p=0.26)$. However, there was a strong association between preexisting right ventricular systolic dysfunction and all-cause in-hospital mortality $(\mathrm{p}=0.00094)$. After multivariate analysis, preexisting right ventricular systolic dysfunction continued to be an independent risk factor for all-cause in-hospital mortality with an odds ratio of 18.9 (95\% Cl: 1.8-201.7; $p=0.015)$.

Conclusion: In this retrospective study of high-risk patients undergoing nonemergent open abdominal surgery, preexisting right ventricular systolic dysfunction was found to have a strong association with allcause in-hospital mortality.

Keywords: Preexisting right ventricular systolic dysfunction, open abdominal surgery, major adverse cardiac events, mortality.

\section{Background}

Major adverse cardiac events (MACE) following non-cardiac surgery have significant implications for morbidity and mortality[1-3]. Although various definitions exist for MACE, it often includes non-fatal cardiac arrest, myocardial infarction, development of congestive heart failure, cerebrovascular event such as stroke, and cardiovascular mortality[4-6]. The revised cardiac risk index (RCRI) is a commonly used tool to predict perioperative cardiac events in patients undergoing noncardiac surgery[7]. In addition, the RCRI has also been shown to have prognostic value for morbidity and mortality in various noncardiac surgery[8]. While ischemic heart disease and congestive heart failure are two cardiovascular diseases included in RCRI as clinical risk factors, the associated left or right ventricular dysfunction is not part of the risk indices[9].

Currently, it is still controversial whether left ventricular (LV) dysfunction, systolic or diastolic, poses as an independent risk factor for perioperative morbidity and mortality in patients undergoing noncardiac 
surgery[10-14]. Moreover, knowledge about the predictive value of right ventricular (RV) dysfunction in noncardiac surgical cohort is largely unknown. Recently, we published the findings that among high-risk patients undergoing major vascular surgery, preexisting RV systolic dysfunction was more predictive of postoperative MACE than LV systolic dysfunction. Indeed, our group found that while left ventricular ejection fraction (LVEF) was not an independent risk factor for MACE, presence of preexisting RV systolic dysfunction by itself was associated with six-folds increase in incidence of postoperative MACE[15].

Building on other authors' previous findings in which the RCRI score was shown to have predictive value for morbidity and mortality in patients undergoing major abdominal procedures[16], and in which LVEF was not an independent risk factor for worse overall outcomes[17], we aimed to elucidate whether the effect of preexisting RV systolic dysfunction on postoperative cardiac morbidity and overall mortality would be similar to what we observed in our previous study. Our hypothesis was that preexisting RV systolic dysfunction would be more prognostic than LV systolic dysfunction for postoperative major adverse cardiac events. As a secondary outcome, we also hypothesized that preexisting RV systolic dysfunction would be associated with higher all-cause in-hospital mortality in major abdominal surgery.

\section{Methods}

This study was approved by the Institutional Review Board at the University of California Irvine Medical Center (UCI IRB HS\# 2017-4099).

\section{Data Collection}

We performed a retrospective single-centered chart review of all patients undergoing open abdominal surgery between 2010 and 2017 (Figure 1). Inclusion criteria for the search were any non-emergent open abdominal procedures including: gastrointestinal procedure (colorectal, gastric, small bowel, hepatic, and pancreatic involvement), urological procedure including nephrectomy or cystectomy, renal transplant, general exploratory laparotomy with or without lysis of adhesion, open removal of retroperitoneal mass, gynecological procedures involving open hysterectomy with or without oophorectomy, open gynecologiconcological tumor debulking, and all other open abdominal surgeries that involved a combination of the procedures mentioned above. Procedures that involved major vascular surgery such as involvement of the abdominal aorta or inferior vena cava were not included in this cohort. The study only included adult patients between the age of 18 and 89. We defined high-risk patients as those who were identified as American Society of Anesthesiologist (ASA) Physical Status Classification of III or IV. Finally, we included only patients with a preoperative echocardiogram performed within one-year of the indexed surgery and for which the study report included evaluation and determination of the RV function. In patients who had multiple echo studies within one-year of the indexed surgery, we selected the study that was closest to the indexed surgery for final review.

To collect patients' demographic and perioperative data, we performed manual chart review using the hospital's electronic record. While intraoperative variables were collected from Surgical Information Systems (Surgical Information Systems Corp, Alpharetta GA), demographic and postoperative variables 
were obtained from Quest (Allscripts Corporation, Alpharetta GA). For those who met the inclusion criteria, the following preoperative variables were collected: age, gender, body mass index (BMI), preoperative hemoglobin level, presence or absence of history of congestive heart failure (CHF), coronary artery disease (CAD), hypertension (HTN), cerebrovascular accident (CVA), diabetes (DM), chronic obstructive pulmonary disease (COPD), obstructive sleep apnea (OSA), and pulmonary hypertension. Since abdominal procedures can often be performed as part of a cancer treatment plan, presence of active cancer requiring the indexed abdominal surgery was also collected. Finally, an RCRI score was calculated and collected based on presence of its six clinical predictors[18].

For intraoperative variables, length of surgery, need for intraoperative transfusion of allogeneic blood products, intraoperative total fluid balance, intraoperative hypotension, and intraoperative infusion of inotropic or vasopressor agents were collected. Furthermore, postoperative variables including postoperative development of respiratory complications and acute kidney injury, as well as, postoperative infections and need for subsequent surgeries during the same admission were also collected so they could be evaluated as confounding factors. Finally, diagnosis of sepsis made anytime during the entire hospital admission was also collected and evaluated as a confounder since presence of sepsis continues to be an important risk factor for morbidity and mortality in surgical patients[19].

For assessment of intraoperative hypotension, both noninvasive and invasive blood pressure measurements were extracted from the Surgical Information System (SIS). Data points without both a systolic and diastolic value were excluded. In addition, any systolic values outside of $20-300 \mathrm{mmHg}$ and diastolic values outside of $5-200 \mathrm{mmHg}$ were excluded as they were considered to be nonphysiological[20]. Blood pressure data from the noninvasive and invasive monitor were then combined in the following manner: if a systolic or diastolic value had another observation of the same type (systolic or diastolic) regardless of the source (noninvasive or invasive) and was within one minute of each other, the two values would be replaced with the average of the two. Mean arterial pressure (MAP) was calculated for each systolic/diastolic pair according to the following equation: $1 / 3 \times$ systolic blood pressure $+2 / 3 x$ diastolic blood pressure. Intraoperative hypotension was defined using MAP $<60 \mathrm{mmHg}$ as the threshold. This threshold was chosen because previous studies have been able to show an increased risk for myocardial injury and mortality when MAP is less than an absolute threshold of 60 for various duration during general surgery[20]. An episode of intraoperative hypotension was derived by calculating area under the threshold (AUT). AUT was calculated in the same manner as previously described by Vernooij et al. [21] Finally, total AUT was obtained by adding all AUTs for each surgical encounter. All blood pressure data processing described was performed via Python version 2.7 using SciPy and NumPy library of packages (Python Software Foundation, Wilmington, DE).

Postoperative MACE was defined broadly as composite events including non-fatal cardiac arrest, myocardial infarction, development of congestive heart failure, cerebrovascular accident (Stroke), and cardiovascular mortality defined as death attributable to any- or a combination of the adverse cardiovascular events just described[4-6]. Post-operative respiratory complication was defined as prolonged intubation for more than 24 hours or need for re-intubation or tracheostomy. Post-operative 
acute kidney injury was defined as patients with a post-operative rise of creatinine greater than $60 \%$ from the baseline[22]. Post-operative need for subsequent surgeries included all procedures that required anesthesia care. Post-operative infection was defined as a composite event including wound or surgical site infection, urinary tract infection, pulmonary infection, and systemic infection. Finally, diagnosis of sepsis was made according to guidelines set by the International Sepsis Definitions Conference[23].

The preoperative echocardiogram obtained within one year of the index surgery was used to identify patients with RV systolic dysfunction. All of the echo studies were originally performed by the cardiology service at the study institution. The majority of the echo studies were performed via transthoracic echocardiogram (96\%). All of the echo images were interpreted by the cardiologist from the study institution with the final results reported and stored in the institution's cardiovascular imaging database (Syngo Dynamics - Siemens Healthcare, Tarrytown, NY). All study reports were reviewed and the following collected: LVEF, right ventricular systolic pressure (RVSP), any valvular pathology categorized as severe, presence of LV diastolic dysfunction, and RV function. RV function was reported as a binary variable (normal versus abnormal). RV function collected from the official report was determined based on visual estimation by the cardiologists. Visual estimation of the RV function was determined based on multiple acoustic windows including apical 4-chamber (lateral wall of the RV and RV apex), parasternal short-axis (anterior, lateral, and inferior wall of the RV), parasternal RV inflow (anterior and inferior wall of the RV), and subcostal 4-chamber (inferior wall of the RV).

\section{Statistical Analysis}

All statistical analysis was performed using SPSS for windows version 24 (SPSS Inc, Chicago, IL). The cohort was divided into 2 groups: those with and without RV systolic dysfunction. For comparative analysis, Fisher's exact test was used for dichotomous variables while Student t-test or Mann-Whitney U test were used for continuous variables with normal and non-normal distribution respectively. For test of normality, Shapiro-Wilk test was employed. Dichotomous variables are reported as counts and percentages while continuous variables are described as either mean and standard deviation for normal distribution or median with interquartile range for non-normal distribution.

Logistic regression analysis was performed to estimate odds ratio (OR) and $95 \%$ confidence interval (Cl) for effect of RV systolic dysfunction on binary outcomes. The selection of variables to include in the univariable logistic model was based on both group differences and a priori predictors. Variables that were individually associated with outcome of interest with $p$-value $<0.1$ in univariable analysis were further included into multivariable analysis in a step-wise manner. Since RV systolic dysfunction, $\mathrm{CHF}$, and RCRI are highly correlated with each other and are expected to exhibit multicollinearity, they were not included in the same regression models during the step-wise multivariable analysis. For goodness-of-fit of the regression model, Hosmer and Lemeshow test was employed. For all tests, a p-value $<0.05$ was considered statistically significant. 


\section{Results}

A total of 122 patients met final inclusion criteria and were included for data analysis (Fig 1). $5.7 \%(\mathrm{~N}=7)$ of the patients in this cohort had preexisting RV systolic dysfunction evident on preoperative echocardiogram at the time of surgery. A comparison of demographic data in patients with and without RV systolic dysfunction showed that there was no difference in gender, age, and BMI between the groups (Table 1). For other preoperative covariates, a higher percentage of patients with RV systolic dysfunction had a history of CAD and CHF ( $p=0.0098$ and $p=0.00052$ respectively). In addition, a higher percentage of patients with RV systolic dysfunction had an RCRI score $>3(p=0.00072)$. For the remaining preoperative covariates such as history of COPD, OSA, hypertension, pulmonary hypertension, and preoperative hemoglobin level, no differences were found between the groups (Table 1). Similarly, there was no statistical differences between the groups when evaluating other echo parameters such as diastolic dysfunction, LVEF, and right ventricular systolic pressure (RVSP). In regards to valvular pathology, only 2 patients had severe valvular pathology (mitral regurgitation and aortic stenosis). Both patients had normal RV function.

For intraoperative covariates, all patients underwent general anesthesia with or without epidural catheter. A higher proportion of patients with RV systolic dysfunction received inotropic or vasopressor infusion during surgery compare to those without RV systolic dysfunction $(p=0.014)$. There was no difference in area under the threshold (AUT) for MAP $<60 \mathrm{mmHg}$ in the analysis of intraoperative hypotension $(p=0.41)$. Moreover, no differences were found between the groups for both intraoperative fluid balance, need for transfusion of blood products, and total surgical time (Table 2). A total of 7(5.7\%) patients developed postoperative acute kidney injury: all 7 patients belonged to the normal RV group $(p=1.00)$. A total of $31(25 \%)$ patients had postoperative respiratory complications. Of those patients with normal RV function, $23.5 \%$ developed postoperative respiratory complications. Among patients with preexisting RV systolic dysfunction, $57.1 \%$ had postoperative respiratory complications $(p=0.070)$.

\section{Complications}

A total of 5 (4.1\%) patients developed postoperative MACE: 3.5\% among patients with normal RV function and $14.3 \%$ among patients with abnormal RV function $(p=0.20) .2$ patients had postoperative myocardial infarction with elevated troponin while 3 patients had postoperative development of heart failure. Neither RV systolic dysfunction nor LVEF were found to be associated with higher incidence of MACE in univariable analysis. An RCRI score $>3$ on the other hand was found to be associated with higher incidence of MACE ( $p=0.0030)$. In addition, CHF and CAD were also found to be associated with higher incidence of MACE ( $p=0.012$ and $p=0.021$ respectively). Multivariable analysis was not carried out due to expected multicollinearity among MACE, CHF, and CAD (Table 3).

A total of 7 (5.7\%) patients had expired during the hospital stay: $3.5 \%$ among patients with normal RV function and $42.9 \%$ among patients with abnormal RV function ( $p=0.0037)$. 1 patient died from sepsis resulting from ischemic bowel, 2 patients died from multi-organ failure relating to sepsis, 1 patient died from protracted course relating to organ rejection after renal transplant, 1 patient died from protracted 
hospital course relating to severe gastrointestinal bleed, 1 patient died from decompensated heart failure, and 1 patient died from multi-organ failure relating to metastatic cancer. There were no intraoperative deaths; all deaths occurred postoperatively during hospital admission.

In univariate logistic regression, the odds ratio (OR) for all-cause in-hospital mortality in presence of preexisting RV systolic dysfunction was 20.8 (95\% Cl, 3.4-125.8; $p=0.00094)$. Other covariates found to have significant association with all-cause in-hospital mortality included sepsis, MACE, CHF, RCRI>3, and postoperative development of respiratory complications (Table 3). Accounting for all other covariates, RV systolic dysfunction remained independently associated with higher incidence of all-cause in-hospital mortality with an $\mathrm{OR}=18.9(95 \% \mathrm{Cl}, 1.8-201.7 ; \mathrm{p}=0.015)$ (Figure 2). RCRI $>3$ was not included in the same regression model as RV systolic dysfunction because of expected multicollinearity. Evaluating RCRI 3 in a separate multivariable regression model, unlike RV dysfunction, it was not an independent risk factor for all-cause in-hospital mortality (Table 3).

\section{Discussion}

In this retrospective single-centered study, we evaluated the association of preexisting RV systolic dysfunction with postoperative major adverse cardiac event (MACE) and all-cause in-hospital mortality in high-risk patients undergoing open abdominal surgery. We found that while preexisting RV systolic dysfunction was not shown to be associated with postoperative MACE in this surgical cohort, it was shown to be associated with higher all-cause in-hospital mortality with a nearly 20 -fold increase in the odds ratio for risk. Similar to previous findings by Gundes et al.[17], we did not find LV systolic dysfunction defined by LVEF to be associated with either outcomes of interest.

In regards to postoperative MACE, its low incidence rate (4.1\%) together with the study's small sample size likely result in the study being underpowered to detect the true effect of preexisting RV systolic dysfunction on postoperative MACE. In regards to all-cause in-hospital mortality, despite the low sample size in the RV dysfunction group, the finding of a strong association between preexisting RV systolic dysfunction and mortality may have clinical significance. First, it has been shown that among patients in the intensive care unit with diagnosis of sepsis, $47 \%$ has isolated RV dysfunction and $53 \%$ has combined biventricular dysfunction evident on echocardiogram[24]. In our surgical cohort, 3 of the 7 patients ultimately died from sepsis. Among these three patients, one had preexisting RV dysfunction. It is possible that development of sepsis in this patient exacerbated the ventricular function in an already compromised RV. Since the RV plays a critical role in delivering deoxygenated blood to the lungs, maintaining forward flow from venous return thereby preventing organ congestion, as well as global systemic circulatory hemostasis[25], it is reasonable to assume that a preexisting RV dysfunction in a septic patient may contribute to increased mortality.

Second, from clinical experience, management of critically-ill patients with concomitant RV systolic dysfunction can be quite difficult. Aside from addressing the underlying problems, optimization of RV function in a patient with preexisting RV dysfunction is challenging. Part of this challenge results from 
the fact that an already compromised RV, unlike that of a healthy RV, is not only sensitive to pressure overload, but also exquisitely sensitive to volume loading. In other words, while preload may be important for optimal RV function, the safety margin in which the rate of fluid replacement and total volume given without further exacerbating ventricular function can be quite narrow in a dysfunctional RV. Combining this challenge with the frequent hemodynamic derangement of hypotension resulting from postsurgical bleed, fluid shift, or in setting of sepsis, the clinical problem and management often becomes much more complex[26].

In addition to the challenge of when and how to optimally replace fluid in a patient with RV systolic dysfunction, avoiding factors that can worsen pulmonary vascular resistance may be just as difficult in a critically-ill patient. This is because a postsurgical and critically-ill patient is often faced with multitude of clinical problems that have negative effect on pulmonary vascular resistance and therefore RV function. These problems may include physiological changes relating to surgically induced stress[27], pulmonary complication resulting in hypoxemia or hypercarbia, mechanical ventilatory setting that increases intrathoracic pressure, or systemic hypotension that requires use of vasoconstrictor agents[28]. In a critically-ill patient with preexisting RV systolic dysfunction, any one or a combination of the above processes would undoubtedly add complexity to the clinical care of the patient. In summary, sepsis may play a role in worsening RV or biventricular function; in a critically-ill patient with preexisting RV systolic dysfunction, development of sepsis may therefore further exacerbate ventricular function and contribute to mortality. Moreover, preexisting RV systolic dysfunction in a postsurgical and critically-ill patient can be particularly challenging and may also contribute to overall in-hospital mortality.

Different from the study performed by Jakobson et al.[16], in which RCRI>3 was evaluated for in-hospital mortality, as well as both short- and long-term mortality, our group only investigated the association between RCRI>3 and all-cause in-hospital mortality. In agreement with this prior study[16], our multivariable analysis did not find RCRI $>3$ to have independent association with all-cause in-hospital mortality. This finding is consistent with the understanding that while RCRI predicts perioperative cardiac events well, it does not reliably predict death in noncardiac surgical cohort[29].

\section{Limitations}

While the findings in this study may have clinical implications and were strongly statistically significant, the sample size was too small. Finding agreement with these results in a larger surgical cohort would be reassuring that they are not a fluke. In addition, the study has other important limitations. First, the retrospective nature of the study means that the quality of the study findings is dependent on accuracy of medical charting. Since our finding of a mortality rate of $5.7 \%$ is comparable with prior studies, this suggests that quality of the study was not likely compromised[30-32]. In addition, the retrospective nature of the study allowed evaluation of well-documented covariates only. As such, there are important a priori variables relevant to this surgical cohort that the study could not account for $[33,34]$.

Second, including preoperative echocardiogram as far as 1 year prior to the indexed surgery may not accurately capture changes in ventricular function. However, the majority of the patients (89\%) had 
preoperative echocardiogram (echo) performed within 6 months of the surgery. Moreover, it is the practice of the institution in which repeat echo is obtained during preoperative evaluation for nonemergent surgery when patient either demonstrate or endorse clinical changes that may have cardiac relevance. With this, we can reasonably assume that the remaining $11 \%$ of the patients in this surgical cohort did not have clinically significant cardiac changes at the time of the surgery.

Lastly, unlike our previous finding in the vascular cohort in which patients with RV systolic dysfunction had lower LVEF and higher RVSP[15], we did not find a significant difference in LVEF and RVSP between patients with and without RV systolic dysfunction in the current surgical cohort. While this may be a reflection of an inherent difference between the vascular versus the abdominal surgical cohort, it may represent a skewed sampling of the population resulting from the study's small sample size. If the latter is true, the study would have additional limitation in that it does not reflect the true epidemiology of patients with RV systolic dysfunction undergoing open abdominal surgery.

\section{Conclusion}

The present study demonstrated that $5.7 \%$ of the patients classified as ASA III and above undergoing nonemergent open abdominal surgery had preexisting RV systolic dysfunction. The presence of RV systolic dysfunction was independently associated with all-cause in-hospital mortality with an almost 20fold increase in odds. LVEF on the other hand, was not associated with overall in-hospital mortality. Based on the study finding, preexisting RV systolic dysfunction appears to be more prognostic for mortality than LV systolic dysfunction in high-risk patients undergoing non-emergent open abdominal surgery. Further studies with larger sample size that includes additional relevant covariates are required to validate current findings.

\section{Abbreviations}

MACE: Major adverse cardiac events

RCRI: Revised cardiac risk index

LV: Left ventricle

RV: Right ventricle

LVEF: Left ventricular ejection fraction

ASA: American Society of Anesthesiology

BMl: Body mass index

CHF: Congestive heart failure 
CAD: Coronary artery disease

HTN: Hypertension

CVA: Cerebral vascular accident

DM: Diabetes

COPD: Chronic obstructive pulmonary disease

OSA: Obstructive sleep apnea

SIS: Surgical information system

MAP: Mean arterial pressure

AUT: Area under the threshold

RVSP: Right ventricular systolic pressure

OR: Odds ratio

Cl: Confidence interval

\section{Declarations}

\section{Ethics Approval and Consent to Participate}

This study was approved by the Institutional Review Board at the University of California Irvine Medical Center (UCI IRB HS\# 2017-4099).

Consent for Publication

Not applicable

Availability of Data and Material

All datasets used and/or analyzed during the current study are available from the corresponding author on reasonable request

Competing Interests

Rinehart is a consultant for Edwards Lifesciences and has ownership interest in Sironis Inc. All other authors do not have any competing interest.

Funding 
This work was supported by Medicine Faculty Research Award, School of Medicine - University of California Irvine.

Authors' contributions

Not applicable

Acknowledgements

Not applicable

\section{References}

1. Kristensen SD, Knuuti J, Saraste A, Anker S, Bøtker H, Hert S, Ford I, Gonzalez-Juanatey J, Gorenek B, Heyndrickx G: Authors/Task Force Members. 2014 ESC/ESA Guidelines on non-cardiac surgery: cardiovascular assessment and management: The Joint Task Force on non-cardiac surgery: cardiovascular assessment and management of the European Society of Cardiology (ESC) and the European Society of Anaesthesiology (ESA). Eur Heart J 2014, 35(35):2383-2431.

2. Sessler DI, Devereaux P: Perioperative troponin screening. Anesthesia \& Analgesia 2016, 123(2):359360.

3. Devereaux P, Sessler DI: Cardiac complications in patients undergoing major noncardiac surgery. New England Journal of Medicine 2015, 373(23):2258-2269.

4. Kip KE, Hollabaugh K, Marroquin OC, Williams DO: The problem with composite end points in cardiovascular studies: the story of major adverse cardiac events and percutaneous coronary intervention. Journal of the American College of Cardiology 2008, 51(7):701-707.

5. Jong M, Worp HB, Graaf Y, Visseren FL, Westerink J: Pioglitazone and the secondary prevention of cardiovascular disease. A meta-analysis of randomized-controlled trials. Cardiovascular diabetology 2017, 16(1):134.

6. Heianza Y, Ma W, Manson JE, Rexrode KM, Qi L: Gut microbiota metabolites and risk of major adverse cardiovascular disease events and death: a systematic review and meta-analysis of prospective studies. Journal of the American Heart Association 2017, 6(7):e004947.

7. Minto G, Biccard B: Assessment of the high-risk perioperative patient. Continuing Education in Anaesthesia, Critical Care \& Pain 2013, 14(1):12-17.

8. Rodseth RN, Biccard BM, Le Manach Y, Sessler DI, Buse GAL, Thabane L, Schutt RC, Bolliger D, Cagini $L$, Cardinale D: The prognostic value of pre-operative and post-operative B-type natriuretic peptides in patients undergoing noncardiac surgery: B-type natriuretic peptide and N-terminal fragment of pro-Btype natriuretic peptide: a systematic review and individual patient data meta-analysis. Journal of the American College of Cardiology 2014, 63(2):170-180.

9. Lee TH, Marcantonio ER, Mangione CM, Thomas EJ, Polanczyk CA, Cook EF, Sugarbaker DJ, Donaldson MC, Poss R, Ho KK: Derivation and prospective validation of a simple index for prediction 
of cardiac risk of major noncardiac surgery. Circulation 1999, 100(10):1043-1049.

10. Karkos CD, Thomson GJ, Hughes R, Hollis S, Hill JC, Mukhopadhyay US: Prediction of cardiac risk before abdominal aortic reconstruction: comparison of a revised Goldman Cardiac Risk Index and radioisotope ejection fraction. Journal of vascular surgery 2002, 35(5):943-949.

11. Sprung J, Abdelmalak B, Gottlieb A, Mayhew C, Hammel J, Levy PJ, O'hara P, Hertzer NR: Analysis of risk factors for myocardial infarction and cardiac mortality after major vascular surgery.

Anesthesiology: The Journal of the American Society of Anesthesiologists 2000, 93(1):129-140.

12. Matyal R, Hess PE, Subramaniam B, Mitchell J, Panzica PJ, Pomposelli F, Mahmood F: Perioperative diastolic dysfunction during vascular surgery and its association with postoperative outcome. Journal of vascular surgery 2009, 50(1):70-76.

13. Karkos C, Baguneid M, Triposkiadis F, Athanasiou E, Spirou P: Routine measurement of radioisotope left ventricular ejection fraction prior to vascular surgery: is it worthwhile? European journal of vascular and endovascular surgery 2004, 27(3):227-238.

14. Arko FR, Hill BB, Olcott IV C, Harris Jr EJ, Fogarty TJ, Zarins CK: Endovascular repair reduces early and late morbidity compared to open surgery for abdominal aortic aneurysm. Journal of Endovascular Therapy 2002, 9(6):711-718.

15. Chou J, Ma M, Gylys M, Seong J, Salvatierra N, Kim R, Jiang L, Barseghian A, Rinehart J: Preexisting Right Ventricular Dysfunction Is Associated With Higher Postoperative Cardiac Complications and Longer Hospital Stay in High-Risk Patients Undergoing Nonemergent Major Vascular Surgery. Journal of cardiothoracic and vascular anesthesia 2018.

16. Jakobson T, Karjagin J, Vipp L, Padar M, Parik A-H, Starkopf L, Kern H, Tammik O, Starkopf J: Postoperative complications and mortality after major gastrointestinal surgery. Medicina 2014, 50(2):111-117.

17. Gündeş E, Aday U, Çiyiltepe H, Çetin DA, Senger AS, Bozdağ E, Gülmez S, Uslu A, Değer KC, Polat E: Effects of left ventricular ejection fraction on morbidity and mortality in major abdominal surgery. Int J Clin Exp Med 2017, 10(12):16632-16638.

18. Eagle KA, Boucher CA: Cardiac risk of noncardiac surgery. In.: Mass Medical Soc; 1989.

19. Moore LJ, Moore FA, Todd S, Jones SL, Turner KL, Bass BL: Sepsis in general surgery: The 2005-2007 national surgical quality improvement program perspective. Archives of Surgery 2010, 145(7):695700.

20. Salmasi V, Maheshwari K, Yang D, Mascha EJ, Singh A, Sessler DI, Kurz A: Relationship between Intraoperative Hypotension, Defined by Either Reduction from Baseline or Absolute Thresholds, and Acute Kidney and Myocardial Injury after Noncardiac SurgeryA Retrospective Cohort Analysis. Anesthesiology 2017, 126(1):47-65.

21. Vernooij L, van Klei W, Machina M, Pasma W, Beattie W, Peelen L: Different methods of modelling intraoperative hypotension and their association with postoperative complications in patients undergoing non-cardiac surgery. British journal of anaesthesia 2018, 120(5):1080-1089. 
22. Khwaja A: KDIGO clinical practice guidelines for acute kidney injury. Nephron Clinical Practice 2012, 120(4):c179-c184.

23. Levy MM, Fink MP, Marshall JC, Abraham E, Angus D, Cook D, Cohen J, Opal SM, Vincent J-L, Ramsay G: $2001 \mathrm{sccm} / \mathrm{esicm} / \mathrm{accp} / \mathrm{ats} /$ sis international sepsis definitions conference. Intensive care medicine 2003, 29(4):530-538.

24. Vallabhajosyula S, Kumar M, Pandompatam G, Sakhuja A, Kashyap R, Kashani K, Gajic O, Geske JB, Jentzer JC: Prognostic impact of isolated right ventricular dysfunction in sepsis and septic shock: an 8-year historical cohort study. Annals of intensive care 2017, 7(1):94.

25. Zochios V, Jones N: Acute right heart syndrome in the critically ill patient. Heart, lung and vessels 2014, 6(3):157.

26. Strunden MS, Heckel K, Goetz AE, Reuter DA: Perioperative fluid and volume management: physiological basis, tools and strategies. Annals of intensive care 2011, 1(1):2.

27. Finnerty CC, Mabvuure NT, Ali A, Kozar RA, Herndon DN: The surgically induced stress response. Journal of parenteral and enteral nutrition 2013, 37(5_suppl):21S-29S.

28. Patil NT: Strategies in patients with right ventricular failure on mechanical ventilation. Indian Journal of Respiratory Care 2018, 7(1):22.

29. Ford MK, Beattie WS, Wijeysundera DN: Systematic review: prediction of perioperative cardiac complications and mortality by the revised cardiac risk index. Annals of internal medicine 2010, 152(1):26-35.

30. Dimick JB, Cowan Jr JA, Upchurch Jr GR, Colletti LM: Hospital volume and surgical outcomes for elderly patients with colorectal cancer in the United States1. Journal of Surgical Research 2003, 114(1):50-56.

31. Ghaferi AA, Birkmeyer JD, Dimick JB: Variation in hospital mortality associated with inpatient surgery. New England Journal of Medicine 2009, 361(14):1368-1375.

32. Noordzij PG, Poldermans D, Schouten O, Bax JJ, Schreiner FA, Boersma E: Postoperative Mortality in The NetherlandsA Population-based Analysis of Surgery-specific Risk in Adults. The Journal of the American Society of Anesthesiologists 2010, 112(5):1105-1115.

33. Bae H-J, Lee H-J, Han D-S, Suh Y-S, Lee Y-H, Lee H-S, Cho J-J, Kong S-H, Yang H-K: Prealbumin levels as a useful marker for predicting infectious complications after gastric surgery. Journal of Gastrointestinal Surgery 2011, 15(12):2136-2144.

34. Sandini M, Pinotti E, Persico I, Picone D, Bellelli G, Gianotti L: Systematic review and meta-analysis of frailty as a predictor of morbidity and mortality after major abdominal surgery. BJS Open 2017 , 1(5):128-137.

\section{Tables}

Table 1 - Baseline Demographic and Comorbidity Data Between Groups 


\begin{tabular}{|l|l|l|l|}
\hline & $\begin{array}{l}\text { Normal Right Ventricular } \\
\text { Function } \\
(\mathrm{n}=115)\end{array}$ & $\begin{array}{l}\text { Right Systolic Ventricular Dysfunction }(\mathrm{n}= \\
7)\end{array}$ & P- value \\
\hline Male & $44(51)$ & $56(4)$ & 0.70 \\
\hline Age & $65[55,74]$ & $56[45,68]$ & 0.13 \\
\hline BMI & $25.4[22.7,30.4]$ & $22.2[20.4,30.3]$ & 0.23 \\
\hline CAD & $22(25)$ & $71(5)$ & 0.0098 \\
\hline CHF & $10(12)$ & $71(5)$ & 0.00052 \\
\hline COPD & $13(15)$ & $29(2)$ & 0.25 \\
\hline OSA & $4(4)$ & $14(1)$ & 0.26 \\
\hline Pulmonary HTN & $15(17)$ & $17(1)$ & 1.00 \\
\hline HTN & $71(81)$ & $43(3)$ & 0.20 \\
\hline $\begin{array}{l}\text { Diastolic } \\
\text { Dysfunction }\end{array}$ & $69(59)$ & $43(3)$ & 1.00 \\
\hline EF & $63(57,67)$ & $62(45,71)$ & 0.66 \\
\hline EF<30\% & $2(2)$ & $14(1)$ & 0.16 \\
\hline RVSP & $32.7(27.2,44.3)$ & $31.3(23.4,64.2)$ & 0.04 \\
\hline Preop Hgb & $10.8(9.6,12.2)$ & $11.2(8.0,11.9)$ & 0.70 \\
\hline Cancer & $50(58)$ & $43(3)$ & $14(1)$ \\
\hline RCRI>3 & $6(7)$ & $57(4)$ & 0.00072 \\
\hline Smoking & $47(43)$ & & \\
\hline
\end{tabular}

Abbreviation: $\mathrm{BMI}=$ body mass index, $\mathrm{CAD}=$ coronary artery disease, $\mathrm{CHF}=$ congestive heart failure, $\mathrm{COPD}=$ chronic obstructive pulmonary disease, OSA = obstructive sleep apnea, Pulmonary HTN = pulmonary hypertension, HTN = hypertension, EF = ejection fraction, RVSP = right ventricular systolic pressure, Preop Hgb = preoperative hemoglobin, RCRI = revised cardiac risk index.

All categorial variables are reported as \% (n). All continuous variables exhibit non-normal distribution; as such, they are reported as median [25th, 75th]

Table 2 - Intraoperative and Post-Operative Variables 


\begin{tabular}{|l|l|l|l|}
\hline & $\begin{array}{l}\text { Normal Right Ventricular } \\
\text { Function }\end{array}$ & $\begin{array}{l}\text { Right Ventricular Systolic } \\
\text { Dysfunction }\end{array}$ & $\begin{array}{l}\text { P- } \\
\text { value* }\end{array}$ \\
\hline Inotrope & $16(13.9)$ & $4(57.1)$ & 0.014 \\
\hline Intraoperative Blood Transfusion & $52(45.2)$ & $3(42.8)$ & 1.00 \\
\hline AUT & $27.1[0,82.6]$ & $61.4[2.6,81.1]$ & 0.41 \\
\hline $\begin{array}{l}\text { Intraoperative Fluid Balance } \\
\text { (mL) }\end{array}$ & $1700[898,2955]$ & $825[170,2000]$ & 0.33 \\
\hline $\begin{array}{l}\text { Surgical Time (minutes) } \\
\text { (median, 25\%, 75\%) }\end{array}$ & $309[194,450]$ & $332[268,690]$ & 0.35 \\
\hline Death & $4(3.5)$ & $3(42.9)$ & 0.0037 \\
\hline MACE & $4(3.5)$ & $1(14.3)$ & 0.26 \\
\hline AKI & $7(7.7)$ & $0(0)$ & 1.00 \\
\hline $\begin{array}{l}\text { Postoperative Respiratory } \\
\text { Complication }\end{array}$ & $27(23.5)$ & $4(57.1)$ & 0.07 \\
\hline
\end{tabular}

Abbreviations: AUT = Area under threshold; MACE = Major adverse cardiac event; AKI = Acute Kidney Injury

*p-value calculated by chi-square for counts and by Mann-Whitney U for scalar data.

Table 3 - Results of Logistic Regression Models for MACE and All-Cause In-Hospital Mortality

\begin{tabular}{|c|c|c|c|c|c|c|}
\hline Model (MACE) & OR & $\mathrm{P}$-value & $95 \% \mathrm{CI}$ & OR & $\mathrm{P}$-value & 95\% CI \\
\hline \multicolumn{4}{|l|}{ Univariate Regression } & \multicolumn{3}{|c|}{ Multivariate Regression } \\
\hline RVD & 4.63 & 0.20 & $(0.45,48.02)$ & \multirow{6}{*}{\multicolumn{3}{|c|}{$*$ Not performed }} \\
\hline Sepsis & 3.41 & 0.30 & $(0.34,34.18)$ & & & \\
\hline $\mathrm{CHF}$ & 11.04 & 0.012 & $(1.69,71.91)$ & & & \\
\hline CAD & 14.00 & 0.021 & $(1.50,130.75)$ & & & \\
\hline EF & 0.97 & 0.52 & $(0.91,1.03)$ & & & \\
\hline RCRI > 3 & 18.56 & 0.0030 & $(2.70,127.73)$ & & & \\
\hline Model (Mortality) & OR & P-value & $95 \% \mathrm{CI}$ & $\mathrm{OR}$ & $\mathrm{P}$-value & $95 \%$ CI \\
\hline \multicolumn{4}{|l|}{ Univariate Regression } & \multicolumn{3}{|c|}{ **Multivariate Regression } \\
\hline RVD & 20.81 & 0.00094 & $(3.44,125.76)$ & 18.86 & 0.015 & $(1.76,201.67)$ \\
\hline Sepsis & 13.623 & 0.0027 & $(2.47,75.16)$ & 9.12 & 0.054 & $(0.96,86.21)$ \\
\hline MACE & 14.93 & 0.0081 & $(2.02,110.45)$ & 5.46 & 0.21 & $(0.39,77.11)$ \\
\hline Multiple Surgery & 3.00 & 0.169 & $(0.63,14.35)$ & \multirow{8}{*}{0.40} & & \\
\hline $\mathrm{CHF}^{* * *}$ & 5.41 & 0.038 & $(1.10,26.74)$ & & & \\
\hline $\mathrm{EF}$ & 1.00 & 0.881 & $(0.93,1.07)$ & & & \\
\hline $\mathrm{CAD}$ & 4.56 & 0.056 & $(0.96,21.71)$ & & & \\
\hline Post-infection & 2.30 & 0.293 & $(0.49,10.92)$ & & & \\
\hline Inotrope & 4.32 & 0.070 & $(0.89,21.05)$ & & & \\
\hline Dialysis & 1.62 & 0.673 & $(0.17,15.15)$ & & & \\
\hline Age & 0.99 & 0.833 & $(0.94,1.05)$ & & & \\
\hline RCRI > 3 & 9.09 & 0.0092 & $(1.73,47.90)$ & 6.99 & 0.07 & $(0.89,55.08)$ \\
\hline Respiratory Complication & 21.36 & 0.0055 & $(2.46,185.77)$ & 8.09 & 0.09 & $(0.72,90.47)$ \\
\hline AKI & 2.767 & 0.386 & $(0.277,27.633)$ & & & \\
\hline Cancer & 0.380 & 0.259 & $(0.071,2.037)$ & & & \\
\hline
\end{tabular}


Abbreviation: RVD = right ventricular (systolic) dysfunction, $\mathrm{CHF}=$ congestive heart failure, $\mathrm{CAD}=$ coronary artery disease, $\mathrm{EF}=$ ejection

fraction, $\mathrm{RCRI}=$ revised cardiac risk index, MACE $=$ major adverse cardiac events, $\mathrm{AKI}=$ acute kidney injury, OR = odds ratio, $\mathrm{CI}=\mathrm{confidence}$ interval

*Multivariate logistic regression model was not performed for MACE due to non-significant finding for RVD and multicollinearity among RCRI, CAD, $\& \mathrm{CHF}$

**Only results from the final models are shown for the multivariate logistic regression analysis for all-cause in-hospital mortality. RVD and RCRI>3 were analyzed in separate models due to multicollinearity. Specific step-wise models are provided in the supplemental.

*** CHF was not included in the multivariate logistic regression due to expected multicollinearity with RVD and RCRI.

\section{Figures}




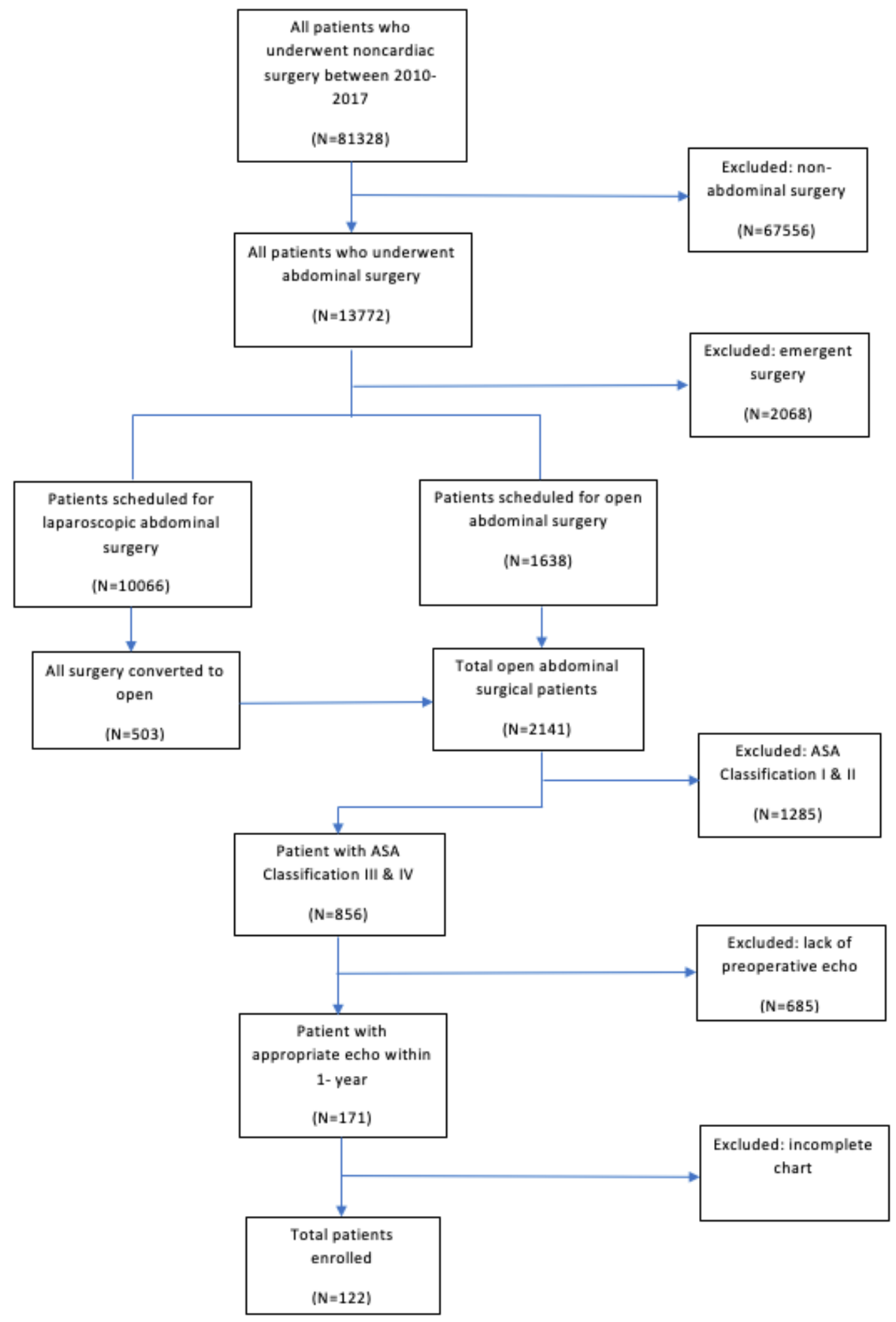

Figure 1

Inclusion and Exclusion Diagram 


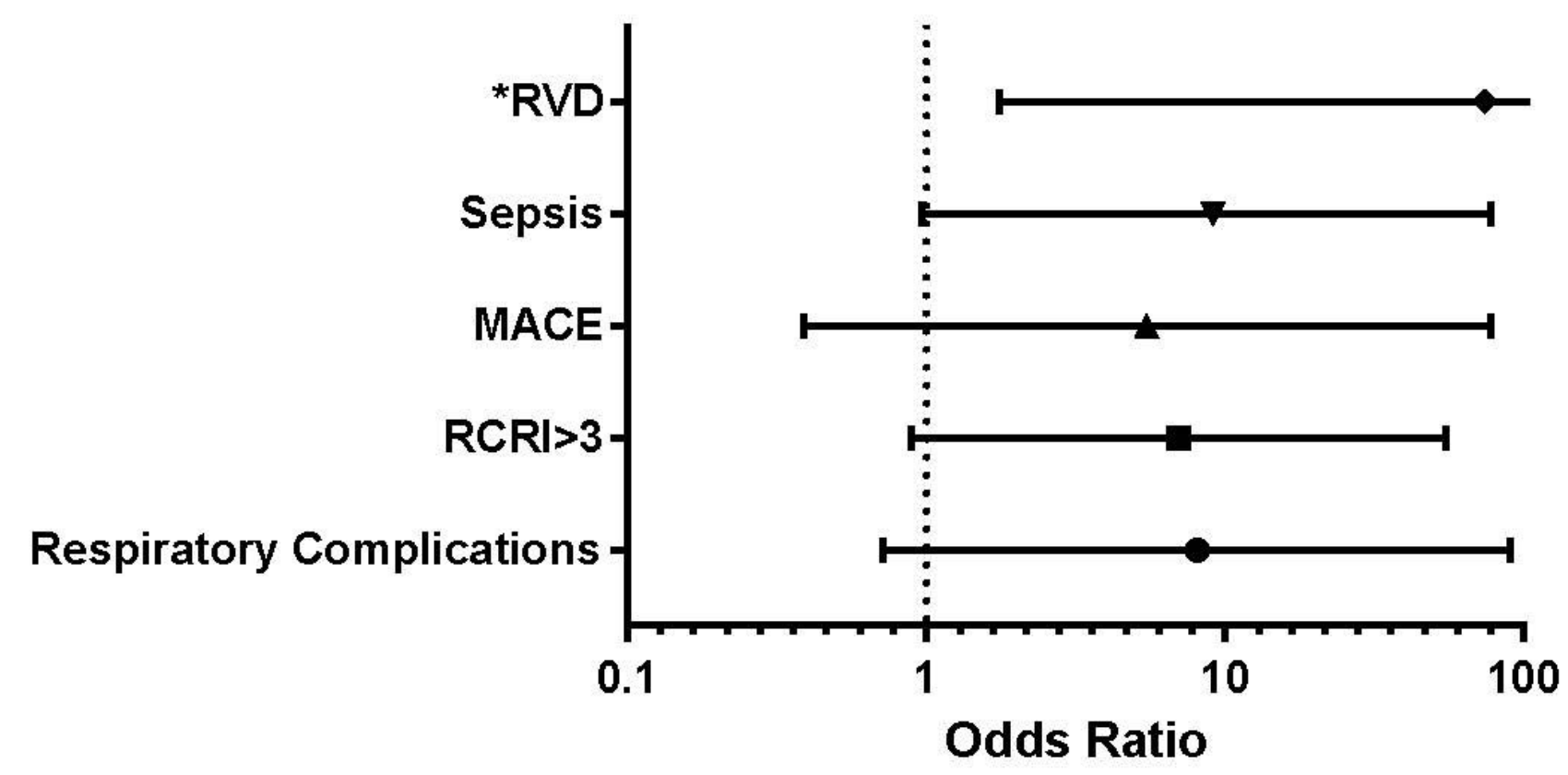

Figure 2

Multivariate Logistic Regression on all-cause in-hospital mortality Abbreviation: RVD = right ventricular (systolic) dysfunction, MACE = major adverse cardiac events, RCRI= revised cardiac risk index *pvalue $<0.05$ 